\title{
The risk of addressing only risks
}

\author{
Garry Egger MPH PhD, John Dixon MBBS PhD
}

See related research article by Manuel and colleagues in CMAJ Open at www.cmajopen.ca/content/2/2/E94

$\mathrm{T}$ he infectious contagions of history strongly underpinned the conditions from which people were most likely to die. Advances in the past century, while aiding in the battle against microbiota, have left a legacy of society-induced chronic diseases. In a linked research paper published in CMAJ Open, Manuel and colleagues ${ }^{1}$ present an analysis of risk factors for heart disease, projected into the future. Their findings predict reduced deaths from cardiovascular disease, which may be timelimited, with changes in the importance of certain risk factors. For example, obesity appears set to take over from smoking as the number one risk factor for cardiovascular death within the next decade. Manuel and colleagues' analysis ${ }^{1}$ describes the relative importance of some risk factors, but it seems that we have yet to fully elucidate all factors that contribute to what has recently been labelled a lifestyle-related disease.

Manuel and colleagues ${ }^{1}$ restricted their analysis to cardiovascular disease and considered some primary associated risk factors (i.e., smoking, body mass index, diabetes, hypertension, elevated blood lipids). However, additional risk factors and determinants have recently been noted. These include excessive alcohol consumption, a sedentary lifestyle, low intake of fruit and vegetables and a high intake of saturated fat. ${ }^{2}$ A recent review ${ }^{3}$ summarized the risk factors responsible for most of the world's chronic disease burden as smoking, excess alcohol consumption, excess weight and dietary factors. The implication being that if all of these factors are modified, all will be well. Such risk factors could account for as much as $60 \%$ of modern chronic diseases, depending on definitions. ${ }^{4}$ However, we require a clearer understanding of the levels of causality and the relative importance of known risk factors to the etiology of modern chronic diseases.

Although infectious diseases are clearly attributable to a microbial cause, establishing causality of noncommunicable diseases is more problematic. The monocausal model of infectious disease etiology has not been adopted in chronic disease epidemiology. In most cases, the agent of causality is ill-defined, although low-grade systemic inflammation (metaflammation ${ }^{5}$ ) has been shown to be a consistent underlying factor. ${ }^{6}$ Different drivers or determinants provide "layers of influence," which link to risk factors or markers and ultimately chronic disease.

This pathway is shown in Figure 1 as levels of determinants, each influencing the next level. Risk factors and markers are most clearly related to metaflammation and chronic diseases, but these, in turn, are influenced by determinants that act proximally or downstream (i.e., more immediate to the disease). These are then influenced by medial and then distal (upstream) factors, indicating not just the cause of the disease, but the cause of the cause and the cause of the cause of the cause?

Epidemiologic analyses usually centre around risk factors (e.g., blood pressure, lipids) and proximal determinants. Somewhat less attention is paid to the more distal determinants that influence lifestyles (e.g., social, political, environmental, economic factors). This focus on what seems to be individual lifestyle choices can lead to victim blaming. For example, the accepted risk factors for heart disease are diabetes, elevated lipids and high blood pressure, while nutritional factors, physical inactivity and smoking are typically considered the main proximal determinants of a range of lifestyle-related chronic conditions. Yet medial determinants are stress (often not considered because of the difficulty of quantification but may contribute to anxiety and depression), peer pressure and social factors. Distal determinants are the "big-picture" concerns of the living environment

\section{- KEY POINTS}

- The burden of chronic disease is often predicted by risk factors, but the environmental upstream determinants of risk factors are complex and challenging to address.

- At a personal level, risk factors for chronic diseases are readily attributed to lifestyle choice and can lead to "victim blaming."

- Artificial environments, their byproducts and the lifestyles they encourage ("anthropogens") are key determinants of chronic disease.

- The price of good population health is ongoing vigilance and managing shifting risk factors and their determinants. 
(e.g., quality and affordability of food, conduciveness to exercise, environmental contaminants) that are often the result of political, economic and social policies; these are rarely considered in clinical epidemiology.

Obesity may, arguably, be more of a marker of problems in the broader society than a direct cause or risk factor. ${ }^{8}$ Alcohol use cannot be excluded, although the hormetic effects of alcohol (small amounts may be beneficial yet large amounts dangerous) and its widespread societal use and promotion, make this a more challenging factor to address from a preventive perspective.

Factors that currently receive little attention but that may come to be important include those related to overexposure to entertainment media, greater access to communications and social media, widespread use of recreational drugs and inadequate sleep. Poor sleep quality is emerging as an underrecognized proximal determinant of conditions from diabetes to heart disease. ${ }^{9}$ Other medial determinants now recognized as potentially important in the causal matrix of noncommunicable diseases include the environment (e.g., exposure to chemicals, pollution, endocrine modifiers, overexposure to sunlight and radiation) and the "invironment" (gut microbial composition). ${ }^{10} \mathrm{We}$ ignore, at our peril, the large-scale observational evidence showing the importance of social determinants of disease, such as social inequity and poor quality of relationships. ${ }^{11}$

It is worth considering an emerging category of potential influences broadly termed "technopathology," which includes the ill-effects of warfare, machinery and vehicle injury on one level, and the rising effects of the overuse of smallscreen technology and social media on the other, resulting in "cyberbullying" and other psychological morbidities to be faced by future generations.

When looking into the future, as Manuel and colleagues $^{1}$ have done, it is important to remember that, throughout history, changes in society have resulted in new patterns of disease epidemiology through changing determinants of health and disease. The modern postindustrial society will be no exception. We have previously used the term "anthropogens" (i.e., artificial environments and byproducts and their associated lifestyles, some which may be detrimental to human health) to define existing and

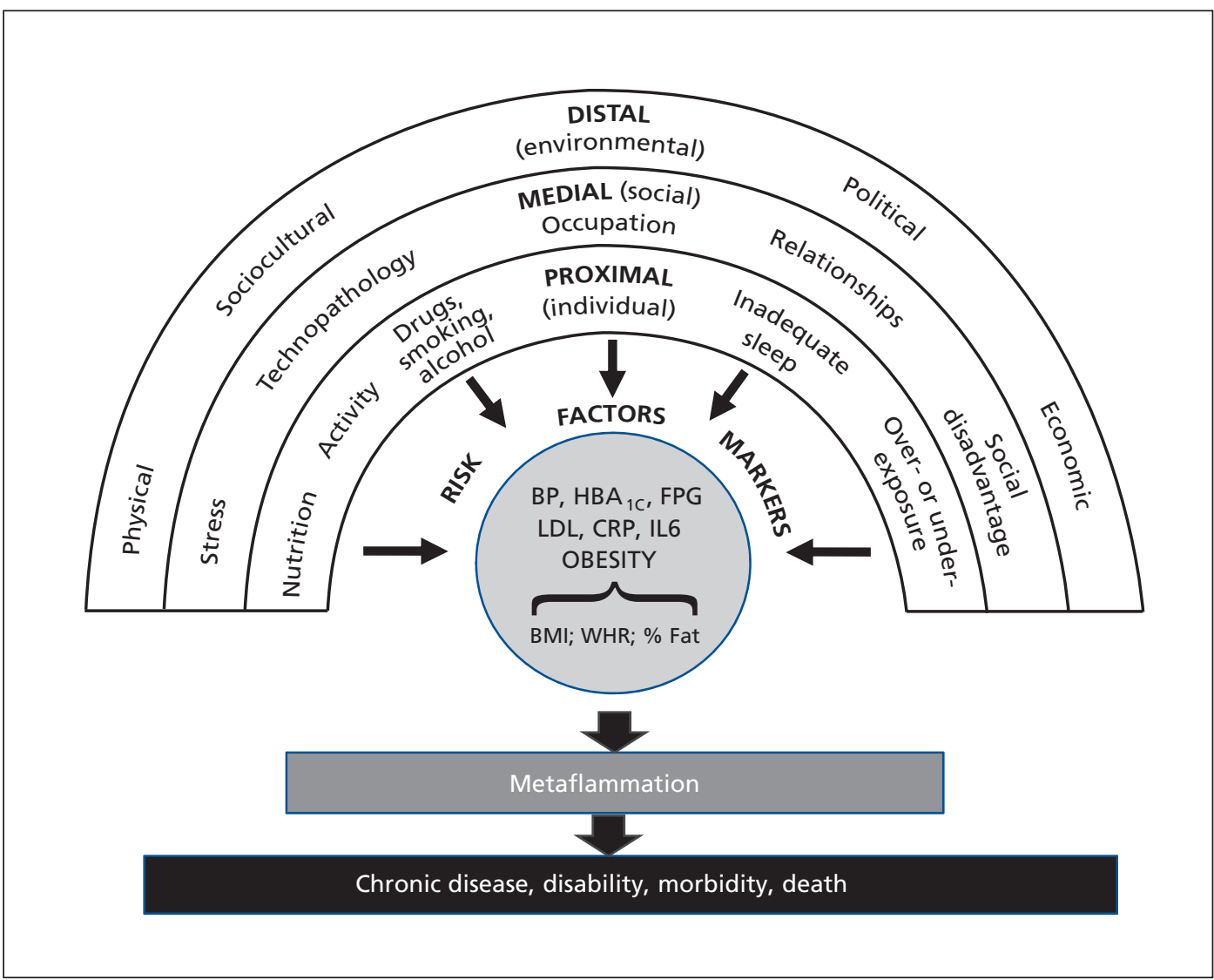

Figure 1: The link between "anthropogens," obesity, metaflammation and chronic disease. Although obesity is often a correlate, this does not necessarily indicate causality. Note: BMI = body mass index, BP = blood pressure, $\mathrm{CRP}=\mathrm{C}$-reactive protein, $\mathrm{FPG}=$ fasting plasma glucose, $\mathrm{HBA}_{1 \mathrm{c}}=$ alpha 1 hemoglobin, IL6 = interleukin 6, LDL = low-density lipoprotein, WHR = waist-to-hip ratio. 
emerging modern determinants of chronic disease. ${ }^{12}$ Reducing traditional risk factors for cardiovascular disease will have an important and relevant role in addressing the growing burden of chronic disease in our communities in the coming years, as shown by Manuel and colleagues. ${ }^{1}$ However, this role is likely to be limited if we do not address the underlying drivers of risks and anticipate and manage changing determinants. The price of good population health is ongoing vigilance and managing shifting risk factors and determinants.

\section{References}

1. Manuel D, Tuna M, Henessy D, et al. Projections of preventable risks for cardiovascular disease in Canada to 2021: a microsimulation modelling approach. CMAJ Open 2013;2;E94-101.

2. Risk factors contributing to chronic disease. Canberra (Australia): Australian Institute of Health and Welfare; 2011. Available: www.aihw.gov.au/publication-detail/?id=10737421466 (accessed 2014 Apr. 25).

3. Ezzati M, Riboli E. Behavioral and dietary risk factors for noncommunicable diseases. N Engl J Med 2013;369:954-64.

4. Chronic diseases and health promotion. Geneva (Switzerland):
World Health Organization; 2014. Available: who.int/chp/en/ (accessed 2014 Apr. 23)

5. Hotamisligil GS. Inflammation and metabolic disease. Nature 2006; $444: 860-7$

6. Libby P. Inflammatory mechanisms: the molecular basis of inflammation and disease. Nutr Rev 2007;65:S140-6.

7. Rose G. The strategy of preventive medicine. Oxford (UK): Oxford University Press; 1992

8. Egger G, Dixon J. Non-nutrient inducers of low grade, systemic inflammation: Support for a "canary in the mineshaft" view of obesity in chronic disease. Obes Rev 2011;12:339-45.

9. Wells ME, Vaughn BV. Poor sleep challenging the health of a Nation. Neurodiagn J 2012;52:233-49.

10. Bäckhed F. 99th Dahlem Conference on infection, inflammation and chronic inflammatory disorders: the normal gut microbiota in health and disease. Clin Exp Immunol 2010;160:80-4.

11. Kiecolt-Glaser JK, Gouin J-P, Hantsoo L. Close relationships, inflammation, and health. Neurosci Biobehav Rev 2010;35:33-8.

12. Egger G. In search of a germ theory equivalent for chronic disease. Prev Chronic Dis 2012;9:E95.

Affiliations: School of Health and Human Sciences (Egger), Southern Cross University, Lismore, New South Wales, Australia; Clinical Obesity Research (Dixon), Baker IDI Heart and Diabetes Institute, Melbourne Australia; Primary Care Research Unit (Dixon), Monash University, Melbourne, Australia.

Contributors: Both of the authors contributed equally to this article and both approved the final version submitted for publication.

\title{
How you can get involved in the CMA!
}

The CMA is committed to providing leadership for physicians and promoting the highest standard of health and health care for Canadians. To strengthen the association and be truly representative of all Canadian physicians the CMA needs to hear from members interested in serving in elected positions and on appointed committees and advisory groups. The CMA structure comprises both governing bodies and advisory bodies either elected by General Council or appointed by the CMA Board of Directors. The Board of Directors - elected by General Council — has provincial/territorial, resident and student representation, is responsible for the overall operation of the CMA and reports to General Council on issues of governance.

CMA committees advise the Board of Directors and make recommendations on specific issues of concern to physicians and the public. Five core committees mainly consist of regional, resident and student representation while other statutory and special committees and task forces consist of individuals with interest and expertise in subject-specific fields. Positions on one or more of these committees may become available in the coming year.

For further information on how you can get involved please go to https://www.cma.ca/en/Pages/get-involved-in-cma.aspx, or contact

\author{
Cherise Araujo \\ Corporate and Governance Services \\ Canadian Medical Association \\ 1867 Alta Vista Drive, Ottawa ON K1G 5 W8 \\ Fax 613 526-7570, Tel $800663-7336$ x1949 \\ cherise.araujo@cma.ca
}

By getting involved, you will have an opportunity to make a difference.

We hope to hear from you!

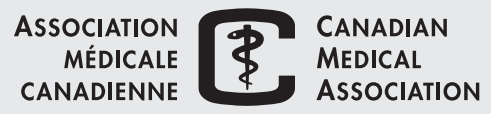

